\title{
A highly efficient, one-pot synthesis of $\alpha$-aminophosphonates over CuO nanopowder
}

\author{
Bikash Karmakar, ${ }^{\text {a }}$ Sanjay Paul, ${ }^{\mathbf{b}}$ and Julie Banerji ${ }^{*}$ \\ ${ }^{a}$ Gobardanga Hindu College, Department of Chemistry, Khantura, North 24 Parganas 743273 , \\ India \\ ${ }^{b}$ Centre of Advanced Studies on Natural products including Organic Synthesis \\ Department of Chemistry, University of Calcutta, 92 A.P.C. Road, Kolkata 700 009, India \\ E-mail: juliebanerji47@gmail.com
}

\begin{abstract}
An atom-efficient, one-pot, three-component synthetic methodology has been developed for the preparation of $\alpha$-aminophosphonates using the crystalline $\mathrm{CuO}$ - nanopowder as catalyst in solvent-free and ambient conditions, for the first time. The reaction involves the use of carbonyl compounds, amines and trimethyl phosphite. A wide range of substrates is compatible in this reaction, producing excellent yields in short time. The catalyst is recyclable with reproducible results.
\end{abstract}

Keywords: $\alpha$-Aminophosphonates, solvent-free reactions, nanopowder, $\mathrm{CuO}$

\section{Introduction}

The classical Kabachnik-Fields reaction involves one- pot three- component coupling of a carbonyl compound, an amine and a phosphite compound, to produce $\alpha$-aminophosphonates. ${ }^{1}$ Aminophosphonates and aminophosphonic acids are considered as the bio- iso-steric phosphorous analogues of $\alpha$-amino acids. ${ }^{2}$ These organophosphorus compounds have drawn the attention of synthetic and medicinal chemists owing to their remarkable biological and pharmacological properties. ${ }^{3}$ The major function of these analogs is displayed through metabolic regulation by inhibiting the metalloenzymes which possess amino acids as substrates. ${ }^{4}$ Moreover, these compounds have been found to be potential antibiotic, ${ }^{5}$ antimicrobial, ${ }^{6}$ and antitumor ${ }^{7}$ agents. They also have several applications in the agricultural industry. ${ }^{8}$ In view of the immense importance of $\alpha$-aminophosphonates researchers in recent years have been engrossed in developing new methodologies for the synthesis of these compounds. ${ }^{9}$ However, many of these reported methods require prolonged reaction time, heating conditions, use of harmful organic solvents, and also involve the use of expensive catalysts which are usually non-recoverable. 
In recent years nanomaterials have attracted a great deal of attention in organic synthesis owing to their high surface- to- volume ratio leading to larger numbers of active sites, and their reusability. Notably, there are few reports available in the literature for the use of non-silicious and non-supported metal oxide nanopowders in multicomponent organic synthesis. ${ }^{10}$ In continuation of our studies towards developing synthetic methods based on nanocatalysts, ${ }^{11}$ we now report, for the first time, an operationally simple, expeditious solvent-free synthesis of $\alpha$ aminophosphonates using highly crystalline $\mathrm{CuO}$ nanopowder under ambient condition (Scheme 1). The large surface area, strong Lewis acid character, and reusable nature of the catalyst has been exploited by us leading to excellent yields of the products in very short reaction times.

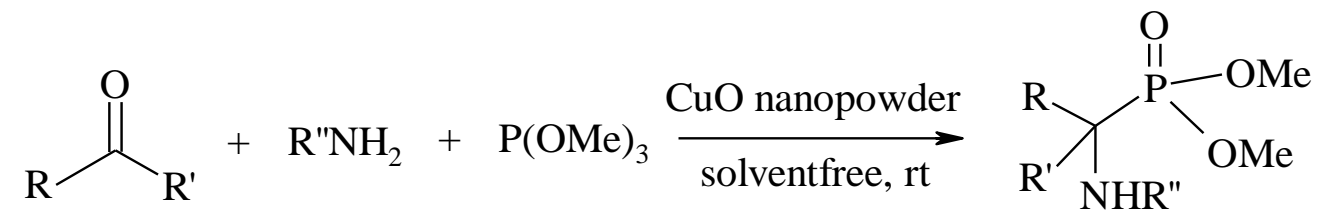

Scheme 1. Synthesis of $\alpha$-aminophosphonates over $\mathrm{CuO}$ nanopowder.

\section{Results and Discussion}

A very simple protocol was followed in the reaction process. A mixture of a carbonyl compound, an amine, and trimethyl phosphite was stirred in the presence of $\mathrm{CuO}$ nanopowder at solvent-free and ambient condition. The catalyst was found to promote the reaction efficiently, affording the corresponding aminophosphonates in high yield. No base or other additives were required in this process. After standard work up, the crude product obtained was passed through a neutral alumina column to isolate the pure compound.

The catalyst was prepared following a non-ionic triblock-copolymer- templated sol-gel approach. ${ }^{12}$ It was characterized by SEM, X-ray diffraction study, and BET surface area analysis. It is evident from the SEM image and X-ray diffraction study of the catalyst that the material is crystalline in nature and the particle sizes are below $50 \mathrm{~nm}$ (Fig. 1). The particles are of almost equal shape and size. To determine the exact crystal planes and particle size, X-ray diffraction analysis was performed. In the wide angle study as shown in Fig. 2, characteristic peaks due to crystal planes appeared, which confirmed its high crystalline character. The peaks appeared to correspond to the (110), (002), (111/200), (202), (020), (202), (113), (311), (220), (311) and (222) planes of monoclinic $\mathrm{CuO}$, which are consistent with standard JCPDS reported values. The FWHM values of the sharp peaks were used to determine the crystallite sizes, applying Sherrer's formula $D_{p}=0.941 \lambda / B \operatorname{Cos} \theta$ where $B$ is the corresponding FWHM value and accordingly, the particle sizes were found to lie between $18-20 \mathrm{~nm}$. The BET method was applied to measure the surface area, which was found to be $101 \mathrm{~m}^{2} / \mathrm{g}$. 

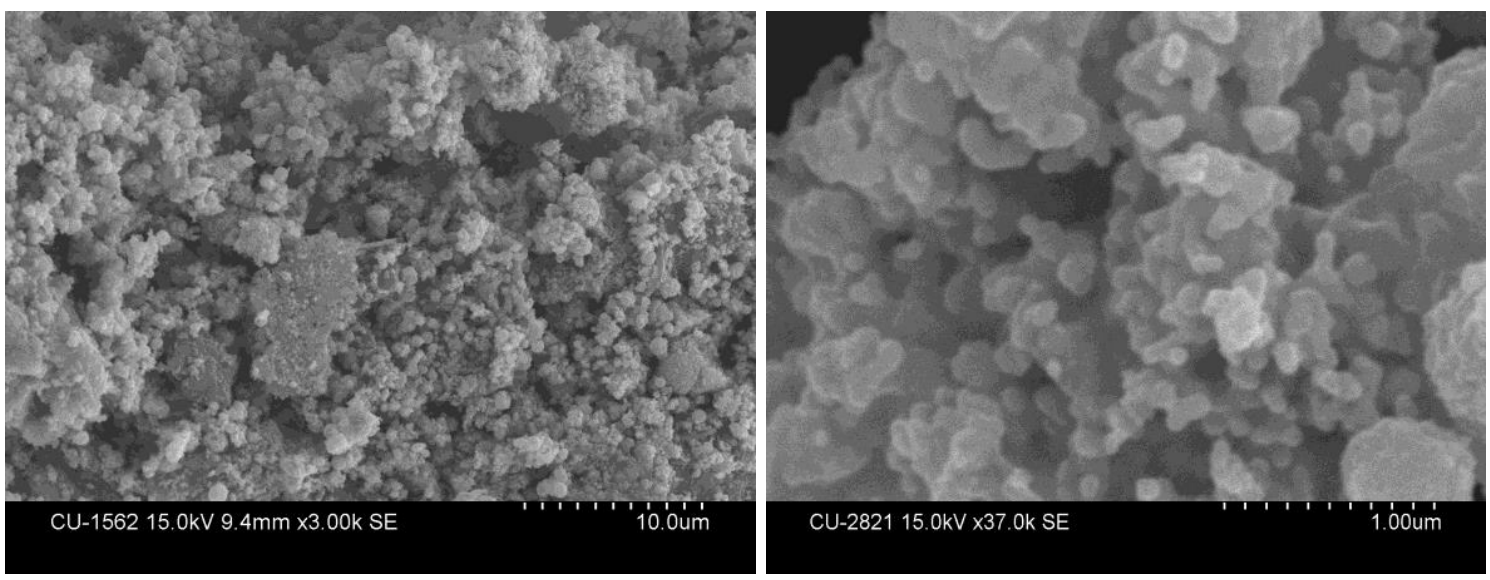

Figure 1. SEM analysis of the $\mathrm{CuO}$ nanopowder.

In our initial study for standardization, a screening was performed with a variety of solvents, taking benzaldehyde, aniline or $\mathrm{P}(\mathrm{OMe})_{3}$ as substrates (Table 1). The use of different conventional organic solvents such as diethyl ether, dichloromethane (DCM), THF, or toluene resulted in low- to moderate yields (entries 1-4). The best result was achieved under solvent-free conditions (entry 5) when the reaction was completed within 1h, in $96 \%$ yield. We also carried out the screening of catalysts using the same reaction. It is pertinent to mention that the reaction hardly proceeded in the absence of catalyst. A variety of $\mathrm{Cu}$ salts was examined in this process. The reaction failed with $\mathrm{Cu}$ granules and $\mathrm{CuO}$ wire (entries 7,8 ). Bulk $\mathrm{CuO}$ and other homogeneous $\mathrm{Cu}$ catalysts such as $\mathrm{CuSO}_{4}$ and $\mathrm{Cu}(\mathrm{OAc})_{2}$ afforded low yields (entries 9-11). High yields were obtained with $\mathrm{CuO}$ - supported mesoporous $\mathrm{SBA} 15$ and $\mathrm{TiO}_{2}$ but the reaction required comparatively longer times for completion (entries 12, 13).

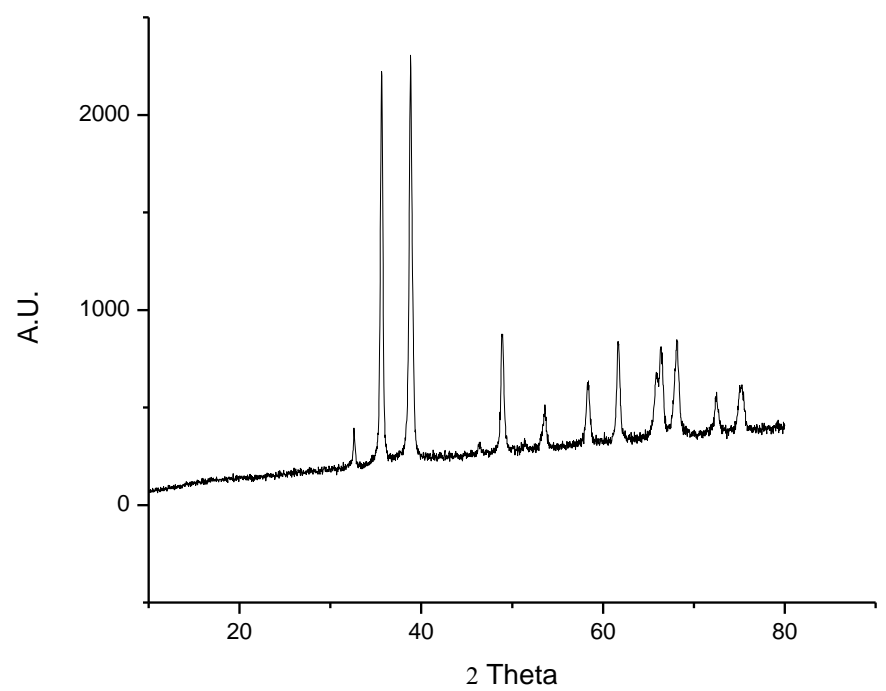

Figure 2. XRD study of the $\mathrm{CuO}$ nanopowder 
After optimizing the reaction conditions, the scope and limitations of the protocol were investigated by treating a diverse range of aldehydes, both aromatic and aliphatic, with aniline and $\mathrm{P}(\mathrm{OMe})_{3}$. The results are summarized in Table 2. Regardless of the nature of the functional groups attached to the aldehydes, all the reactions proceeded smoothly, generating the corresponding $\alpha$-aminophosphonates in good yields (entries 1-8). However, aromatic aldehydes containing hydroxy groups took longer times for completion (entries 9,10). The reaction was successful with aliphatic aldehydes (entries 14,15) which also resulted in high yields. We continued our research to test the reactivity of ketones under similar conditions. However, in this case the reactions were sluggish to afford the corresponding $\alpha$-aminophosphonates. Cyclohexanone and cyclopentanone produced good yields, but with acetophenone the yield was moderate (entries 16-18). We also studied the reaction of different amines, both aromatic and aliphatic, with benzaldehyde and $\mathrm{P}(\mathrm{OMe})_{3}$ (Table 3). In these cases also good conversions were observed within short times. Interestingly, aliphatic amines, viz., cyclohexylamine, pyrrolidine, piperidine, and morpholine, showed faster rates compared to aromatic amines, possibly due to their higher basicity. The formation of $\alpha$-hydroxyphosphonate as a byproduct was not observed in any of the reactions.

Table 1. Optimization of reaction conditions in synthesis of $\alpha$-aminophosphonates ${ }^{\mathrm{a}}$

\begin{tabular}{ccccc}
\hline Entry & Solvent & Catalyst & Time(h) & Yield $(\%)^{\mathrm{b}}$ \\
\hline 1 & Diethyl ether & $\mathrm{CuO} \mathrm{np}$ & 6.0 & 34 \\
2 & Dichloromethane & $\mathrm{CuO} \mathrm{np}$ & 6.0 & 47 \\
3 & Tetrahydrofuran & $\mathrm{CuO} \mathrm{np}$ & 6.0 & 68 \\
4 & Toluene & $\mathrm{CuO} \mathrm{np}$ & 6.0 & 53 \\
5 & Solvent-free & $\mathrm{CuO} \mathrm{np}$ & 1.0 & 96 \\
6 & Solvent-free & None & 12 & - \\
7 & Solvent-free & $\mathrm{Cu} \mathrm{granules}$ & 12 & - \\
8 & Solvent-free & $\mathrm{CuO}$ wire & 12 & - \\
9 & Solvent-free & $\mathrm{Bulk} \mathrm{CuO}$ & 6.0 & 46 \\
10 & Solvent-free & $\mathrm{CuSO} 4.5 \mathrm{H}_{2} \mathrm{O}$ & 6.0 & 67 \\
11 & Solvent-free & $\mathrm{Cu}\left(\mathrm{Oac}_{2} . \mathrm{H}_{2} \mathrm{O}\right.$ & 6.0 & 78 \\
12 & Solvent-free & $\mathrm{CuO} / \mathrm{SBA}_{15}(20)$ & 2.5 & 90 \\
13 & Solvent-free & $\mathrm{CuO} / \mathrm{TiO}_{2}(20)$ & 3.0 & 85 \\
\hline
\end{tabular}

${ }^{a}$ Reaction conditions: 1.0 eq. benzaldehyde, 1.0 eq. amine, 1.3 eq. $\mathrm{P}(\mathrm{OMe})_{3}, 20 \mathrm{mg}$ catalyst, $\mathrm{RT}$ stirring, wt \% loading in parentheses, ${ }^{\mathrm{b}}$ isolated yield.

From the mechanistic point of view we believe that the nano-sized $\mathrm{Cu}{ }^{\mathrm{II}} \mathrm{O}$, an efficient Lewis acid, is the main active site in the reaction. It promotes the formation of imines by condensation of a carbonyl function and amine. The activated imine then reacted with $\mathrm{P}(\mathrm{OMe})_{3}$ to generate the 
corresponding $\alpha$-aminophosphonates. A probable mechanistic pathway has been proposed in Fig. 3. The superior catalytic activity of $\mathrm{CuO}$ nanopowder can be distinctly proved by the comparison of its reactivity with bulk $\mathrm{CuO}$ (entries 5 and 9, table 1). This difference is obviously due to high specific surface area of our catalyst $\left(101 \mathrm{~m}^{2} / \mathrm{gm}\right)$ compared to bulk $\mathrm{CuO}\left(4.1 \mathrm{~m}^{2} / \mathrm{gm}\right)$ thereby generating larger number of active sites.

Table 2. One-pot synthesis of $\alpha$-aminophosphonates with various carbonyl compounds ${ }^{\mathrm{a}}$<smiles>[R]C([R])=O</smiles><smiles>[R]C(Nc1ccccc1)P(=O)(OC)OC</smiles>

1a-o

$\mathbf{2 a}$

3a-o

\begin{tabular}{|c|c|c|c|c|}
\hline Entry & Carbonyl compounds & Product & Time (h) & Yield $(\%)^{\mathrm{b}}$ \\
\hline 1 & $\mathrm{C}_{6} \mathrm{H}_{5} \mathrm{CHO}$ & $\mathbf{3 a}$ & 1.0 & 96 [9j] \\
\hline 2 & $4-\mathrm{O}_{2} \mathrm{~N}-\mathrm{C}_{6} \mathrm{H}_{4} \mathrm{CHO}$ & $\mathbf{3 b}$ & 0.7 & 92 [9i] \\
\hline 3 & $3-\mathrm{O}_{2} \mathrm{~N}-\mathrm{C}_{6} \mathrm{H}_{4} \mathrm{CHO}$ & $3 c$ & 0.5 & 88 \\
\hline 4 & $4-\mathrm{Cl}-\mathrm{C}_{6} \mathrm{H}_{4} \mathrm{CHO}$ & $3 d$ & 0.5 & $91[9 \mathrm{j}]$ \\
\hline 5 & $4-\mathrm{Me}-\mathrm{C}_{6} \mathrm{H}_{4} \mathrm{CHO}$ & $3 e$ & 1.0 & $90[9 \mathrm{i}]$ \\
\hline 6 & $4-\mathrm{CN}-\mathrm{C}_{6} \mathrm{H}_{4} \mathrm{CHO}$ & 3f & 0.7 & $92[9 j]$ \\
\hline 7 & $4-\mathrm{MeO}-\mathrm{C}_{6} \mathrm{H}_{4} \mathrm{CHO}$ & $3 \mathbf{g}$ & 0.5 & $94[9 \mathrm{~h}]$ \\
\hline 8 & $3-\mathrm{MeO}-\mathrm{C}_{6} \mathrm{H}_{4} \mathrm{CHO}$ & $3 h$ & 1.0 & 86 \\
\hline 9 & $4-\mathrm{HO}-\mathrm{C}_{6} \mathrm{H}_{4} \mathrm{CHO}$ & $3 \mathbf{i}$ & 2.0 & $78[9 \mathrm{~h}]$ \\
\hline 10 & 4-HO-3-MeO- $\mathrm{C}_{6} \mathrm{H}_{3} \mathrm{CHO}$ & $3 \mathbf{j}$ & 2.0 & 75 \\
\hline 11 & 3,4-di- $\mathrm{MeO}-\mathrm{C}_{6} \mathrm{H}_{3} \mathrm{CHO}$ & $3 \mathbf{k}$ & 1.0 & 89 \\
\hline 12 & 3,4,5-tri- $\mathrm{MeO}-\mathrm{C}_{6} \mathrm{H}_{2} \mathrm{CHO}$ & 31 & 1.2 & $90[9 r]$ \\
\hline 13 & 1-Naphthaldehyde & $3 m$ & 0.5 & 94 \\
\hline 14 & $i$-PrCHO & $3 n$ & 0.7 & $85[9 \mathrm{~h}]$ \\
\hline 15 & $\mathrm{EtCHO}$ & 30 & 1.0 & 91 \\
\hline 16 & Cyclohexanone & $3 p$ & 4.0 & $85[9 \mathrm{j}]$ \\
\hline 17 & Cyclopentanone & $3 q$ & 6.0 & $76[9 \mathrm{j}]$ \\
\hline 18 & Acetophenone & $3 \mathbf{r}$ & 6.0 & $75[9 \mathrm{t}]$ \\
\hline
\end{tabular}

${ }^{a}$ Reaction conditions: 1.0 eq. benzaldehyde, 1.0 eq. amine, 1.3 eq. $\mathrm{P}(\mathrm{OMe})_{3}, 20 \mathrm{mg}$ catalyst, solvent-free, RT stirring. ${ }^{b}$ Isolated yield, references in parentheses.

The reusable nature of the catalyst was studied through the condensation of benzaldehyde, aniline and $\mathrm{P}(\mathrm{OMe})_{3}$. Under the stabilized condition the reaction was carried out in the presence of nano- $\mathrm{CuO}$ catalyst. After completion of the reaction, the reaction mixture was centrifuged at 
$3000 \mathrm{rpm}$ for $10 \mathrm{~min}$ and the supernatant layer was decanted. The deposited catalyst was collected, followed by washing with acetone several times to remove all the organic substances. It was then dried at $80{ }^{\circ} \mathrm{C}$ for $4 \mathrm{~h}$ and was re-used 4 consecutive times with fresh batches of reactants following the same process. With all the batches the reaction proceeded smoothly, with almost reproducible results each time (Fig. 4)

Table 3. One-pot synthesis of $\alpha$-aminophosphonates with various amines ${ }^{\text {a }}$

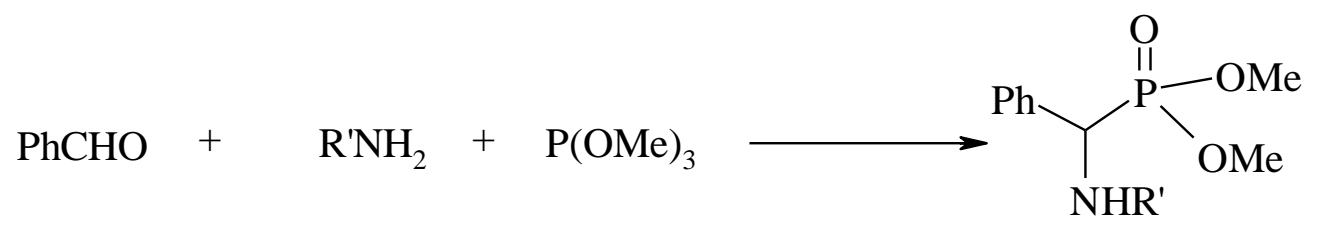

$1 a \quad 2 a-1$

4a-1

\begin{tabular}{ccccc}
\hline Entry & Amine & Product & Time (h) & Yield (\%) \\
\hline 1 & $\mathrm{C}_{6} \mathrm{H}_{5}-\mathrm{NH}_{2}$ & $\mathbf{4 a}$ & 0.5 & $96[9 \mathrm{j}]$ \\
2 & $2-\mathrm{MeC}_{6} \mathrm{H}_{4}-\mathrm{NH}_{2}$ & $\mathbf{4 b}$ & 0.5 & 86 \\
3 & $4-\mathrm{MeC}_{6} \mathrm{H}_{4}-\mathrm{NH}_{2}$ & $\mathbf{4 c}$ & 0.7 & $89[9 \mathrm{~s}]$ \\
4 & $4-\mathrm{ClC}_{6} \mathrm{H}_{4}-\mathrm{NH}_{2}$ & $\mathbf{4 d}$ & 0.5 & $90[9 \mathrm{j}]$ \\
5 & $4-\mathrm{BrC}_{6} \mathrm{H}_{4}-\mathrm{NH}_{2}$ & $\mathbf{4 e}$ & 1.0 & 85 \\
6 & $4-\mathrm{MeOC}_{6} \mathrm{H}_{4}-\mathrm{NH}_{2}$ & $\mathbf{4 f}$ & 1.0 & $81[9 \mathrm{j}]$ \\
7 & $3-\mathrm{O}_{2} \mathrm{NC}_{6} \mathrm{H}_{4}-\mathrm{NH}_{2}$ & $\mathbf{4 g}$ & 1.2 & 85 \\
8 & $\mathrm{C}_{6} \mathrm{H}_{5} \mathrm{CH}_{2}-\mathrm{NH}_{2}$ & $\mathbf{4 h}$ & 0.7 & $80[9 \mathrm{j}]$ \\
9 & $\mathrm{Cyclohexylamine}_{1}$ & $\mathbf{4 i}$ & 1.0 & $87[9 \mathrm{~s}]$ \\
10 & Pyrrolidine $_{11}$ & $\mathbf{4 j}$ & 0.5 & $90[9 \mathrm{e}]$ \\
12 & Piperidine & $\mathbf{4 k}$ & 0.3 & $87[9 \mathrm{e}]$ \\
\hline
\end{tabular}

${ }^{a}$ Reaction conditions. 1.0 eq. benzaldehyde, 1.0 eq. amine, 1.3 eq. $\mathrm{P}(\mathrm{OMe})_{3}, 20 \mathrm{mg}$ catalyst, solvent-free, RT stirring, ${ }^{\mathrm{b}}$ Isolated yield, references in parentheses. 


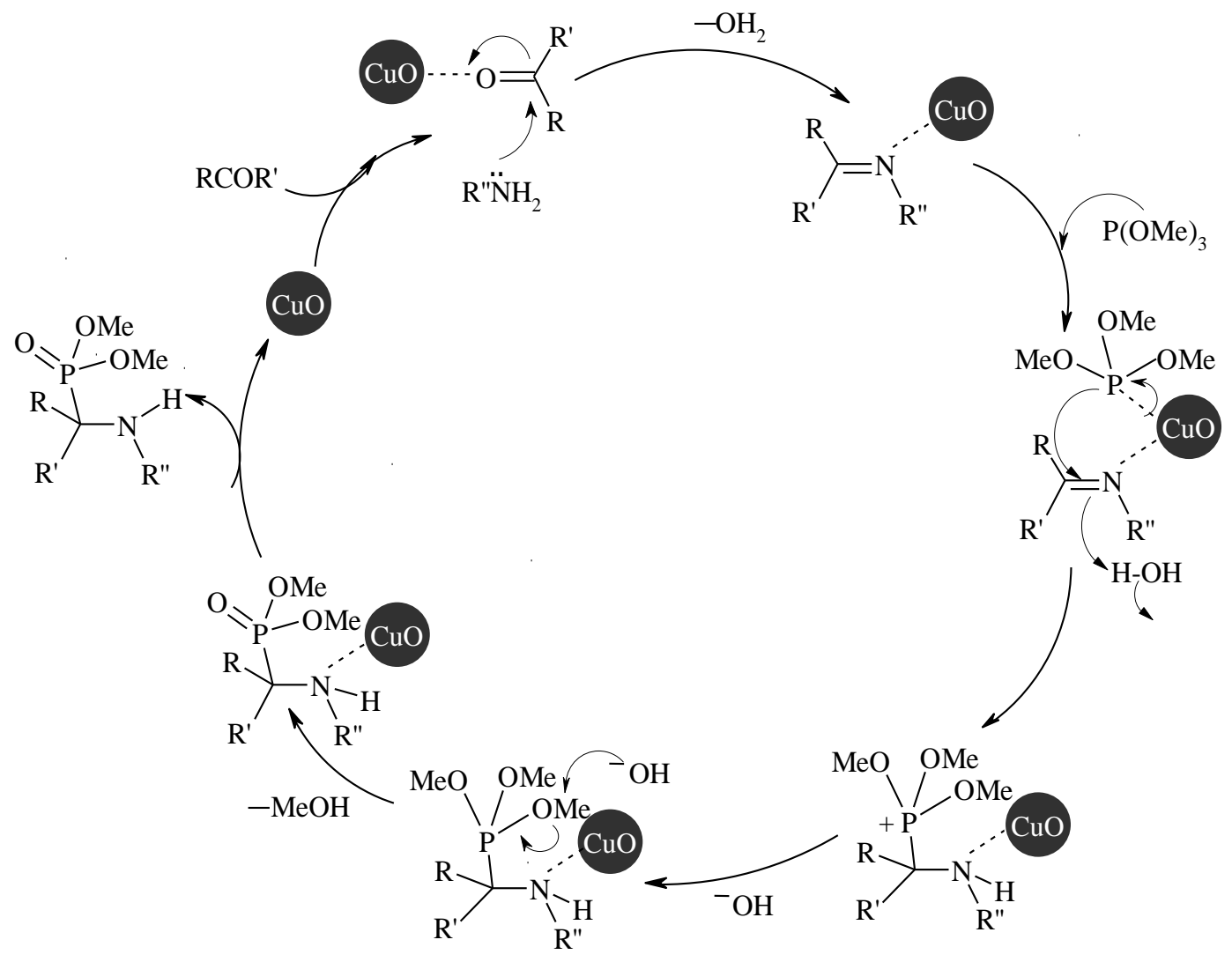

Figure 3. Probable mechanism for the $\mathrm{CuO}$ nanopowder- catalyzed synthesis of $\alpha$ aminophosphonates.

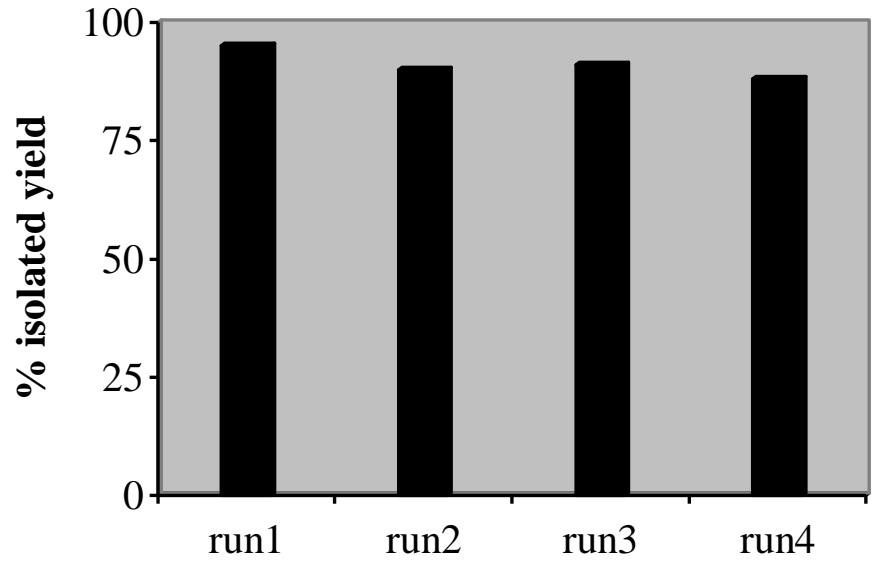

Figure 4. Reusability study of $\mathrm{CuO}$ nanopowder with entry 1, Table 2. 


\section{Experimental Section}

General. ${ }^{1} \mathrm{H}$ - NMR and ${ }^{13} \mathrm{C}$ - NMR spectral analyses were carried out on Bruker-Avance Digital $300 \mathrm{MHz}$ and $75.5 \mathrm{MHz}$ instruments; tetramethylsilane (TMS) was used as internal standard. Infrared spectra were recorded in $\mathrm{KBr}$ pellets in reflection mode on a Perkin Elmer RX-1 FTIR spectrophotometer. SEM images were obtained from a Hitachi S-3400N microscope at an operating voltage of $15 \mathrm{kV}$. The sample was coated with gold for effective imaging before being charged. X-Ray powder diffraction study was carried out on a Philips PW-1830 X-Ray diffractometer at a voltage of $35 \mathrm{kV}$ and current of $25 \mathrm{~mA}$ using $\mathrm{CuK \alpha}$ radiation $(\lambda=154 \mathrm{~nm})$ at a scanning rate of $1 \%$ minute in the $2 \theta$ range $10-80^{\circ}$. Melting points (uncorrected) were determined on a Köfler Block apparatus. E. Merck aluminum-backed silica gel plates coated with silica gel $\mathrm{G}$ were used for analytical TLC and monitored under UV light (254 and $360 \mathrm{~nm}$ ) and also by exposure to iodine vapor. Synthetic grade chemicals from Acros and E-Merck were used for the preparation of the catalyst and from Spectrochem for carrying out the organic reactions. For column chromatography Merck neutral alumina (fine mesh) was used. All solvents used in the reaction were distilled and dried over $\mathrm{Na}_{2} \mathrm{SO}_{4}$. RT denotes room temperature.

\section{Typical procedure for the synthesis of $\mathrm{CuO}$ nanopowder}

The catalyst was synthesized by a sol-gel approach where the non-ionic triblock copolymer P123 $\left(\mathrm{EO}_{20} \mathrm{PO}_{70} \mathrm{EO}_{20}, \mathrm{EO}=\right.$ ethylene oxide, $\mathrm{PO}=$ propylene oxide, $\mathrm{MW}=5800$, Sigma-Aldrich $)$ acted as the structure- directing template. The copolymer $(1.0 \mathrm{~g})$ was dissolved in $10 \mathrm{~mL}$ of anhydrous ethanol at RT to get a clear solution. Then quantitative amount of $\mathrm{Cu}\left(\mathrm{NO}_{3}\right)_{2} \cdot 3 \mathrm{H}_{2} \mathrm{O}(5 \mathrm{mmol}$, EMerck) was added to it and the blue solution was stirred at RT for $2 \mathrm{~h}$. The homogeneous gel was then aged at $40{ }^{\circ} \mathrm{C}$ for $24 \mathrm{~h}$ for slow solvent evaporation of the solvent. It was then dried at 100 ${ }^{\circ} \mathrm{C}$ for $36 \mathrm{~h}$ to obtain a green mass. Finally, it was calcined at $500{ }^{\circ} \mathrm{C}$ for $4 \mathrm{~h}$ in air by slowly increasing the temperature from $\mathrm{RT}$ (ramping rate of $5^{\circ} \mathrm{C} / \mathrm{min}$ ) to provide the black $\mathrm{CuO}$ nanopowder.

\section{Typical procedure for the synthesis of $\alpha$-aminophosphonates}

A mixture of carbonyl compound $(1.0 \mathrm{mmol})$, amine $(1.0 \mathrm{mmol})$, trimethyl phosphite $(1.3 \mathrm{mmol})$ and $20 \mathrm{mg}$ catalyst was stirred at RT in the absence of any solvent for certain periods as indicated in Table 2 and 3. After completion (monitored by TLC), the reaction mixture was diluted with DCM and centrifuged to separate the catalyst for reuse. The organic layer was decanted and added to water. It was then extracted with DCM $(3 \times 10 \mathrm{~mL})$. This extract was dried over anhydrous $\mathrm{Na}_{2} \mathrm{SO}_{4}$ and concentrated under reduced pressure. The crude product was purified by column chromatography using neutral alumina with ethyl acetate/hexane as eluent. All the isolated compounds were characterized by mp, IR, ${ }^{1} \mathrm{H}$ NMR, ${ }^{13} \mathrm{C}$ NMR and elemental analysis $(\mathrm{C}, \mathrm{H}$, and $\mathrm{N})$. Spectral data of the representative new products are given below. 
Dimethyl $\boldsymbol{\alpha}$-( $\boldsymbol{N}$-anilino)- $\boldsymbol{\alpha}$-(3-nitrophenyl)methylphosphonate (3c). Yield $196 \mathrm{mg}$; yellow crystalline solid; mp $98{ }^{\circ} \mathrm{C}$; IR (KBr): 3290, 3033, 1602, 1526, 1349, 1243, 1043, $836 \mathrm{~cm}^{-1} ;{ }^{1} \mathrm{H}$ NMR $\left(\mathrm{CDCl}_{3}, 300 \mathrm{MHz}\right): \delta 3.62(\mathrm{~d}, J=10.8 \mathrm{~Hz}, 3 \mathrm{H}), 3.8(\mathrm{~d}, J=10.8 \mathrm{~Hz}, 3 \mathrm{H}), 4.85-4.97(\mathrm{~m}$, $2 \mathrm{H}), 6.57(\mathrm{~d}, J=7.8 \mathrm{~Hz}, 2 \mathrm{H}), 6.74(\mathrm{t}, J=7.4 \mathrm{~Hz}, 1 \mathrm{H}), 7.12(\mathrm{~d}, J=7.8 \mathrm{~Hz}, 2 \mathrm{H}), 7.53(\mathrm{t}, J=7.9$ $\mathrm{Hz}, 1 \mathrm{H}), 7.83(\mathrm{~d}, J=7.2 \mathrm{~Hz}, 1 \mathrm{H}), 8.15(\mathrm{~d}, J=7.5 \mathrm{~Hz}, 1 \mathrm{H}), 8.34(\mathrm{~d}, J=1.8 \mathrm{~Hz}, 1 \mathrm{H}) ;{ }^{13} \mathrm{C}$ NMR $\left(\mathrm{CDCl}_{3}, 75.5 \mathrm{MHz}\right): \delta 53.8,54.0,55.2,56.0,113.9,114.7,127.32,128.85,128.93,129.14$, 146.1, 159.4; Anal. Calcd for $\mathrm{C}_{15} \mathrm{H}_{17} \mathrm{~N}_{2} \mathrm{O}_{5} \mathrm{P}$ : C, 53.57; H, 5.06; N, 8.33. Found: C, 53.49; H, $5.17 ; \mathrm{N}, 8.45$.

Dimethyl- $\alpha$-( $\boldsymbol{N}$-anilino)- $\boldsymbol{\alpha}$-(3-Methoxyphenyl)methylphosphonate (3h). Yield $201 \mathrm{mg}$; gray white powder; mp $65{ }^{\circ} \mathrm{C}$. IR (KBr): 3308, 2949, 1598, 1493, 1262, 1231, 1033, $753 \mathrm{~cm}^{-1} ;{ }^{1} \mathrm{H}$ NMR $\left(\mathrm{CDCl}_{3}, 300 \mathrm{MHz}\right): \delta 3.51(\mathrm{~d}, J=10.5 \mathrm{~Hz}, 3 \mathrm{H}), 3.78(\mathrm{~d}, J=10.8 \mathrm{~Hz}, 3 \mathrm{H}), 3.80(\mathrm{~s}, 3 \mathrm{H})$, $4.73(\mathrm{~m}, 1 \mathrm{H}), 4.80(\mathrm{~m}, 1 \mathrm{H}), 6.61(\mathrm{dd}, J=3.5 \mathrm{~Hz}, 7.8 \mathrm{~Hz}, 2 \mathrm{H}), 6.68-6.74(\mathrm{~m}, 1 \mathrm{H}), 6.82$ (d, $J=8.1$ $\mathrm{Hz}, 1 \mathrm{H}), 7.02-7.39(\mathrm{~m}, 5 \mathrm{H}) ;{ }^{13} \mathrm{C} \mathrm{NMR}\left(\mathrm{CDCl}_{3}, 75.5 \mathrm{MHz}\right): \delta 53.83,54.71,55.32,56.7,113.39$, $113.46,113.86,118.58,120.14,129.2,129.7,137.24,146.2,159.9 ;$ Anal. Calcd for $\mathrm{C}_{16} \mathrm{H}_{20} \mathrm{NO}_{4} \mathrm{P}: \mathrm{C}, 59.81 ; \mathrm{H}, 6.23$; N, 4.36. Found: C, 59.68; H, 6.15; N, 4.29.

Dimethyl $\boldsymbol{\alpha}$-( $\boldsymbol{N}$-anilino)- $\boldsymbol{\alpha}$-(3,4-dimethoxyphenyl)methylphosphonate (3k). Yield $187 \mathrm{mg}$; white solid; mp $78{ }^{\circ} \mathrm{C}$; IR (KBr): 3293, 3108, 2953, 1601, 1513, 1457, 1241, 1036, $756 \mathrm{~cm}^{-1} ;{ }^{1} \mathrm{H}$ NMR $\left(\mathrm{CDCl}_{3}, 300 \mathrm{MHz}\right): \delta 3.51(\mathrm{~d}, J=9.9 \mathrm{~Hz}, 3 \mathrm{H}), 3.75(\mathrm{~d}, J=10.6 \mathrm{~Hz}, 3 \mathrm{H}), 3.84(\mathrm{~s}, 3 \mathrm{H})$, $3.88(\mathrm{~s}, 3 \mathrm{H}), 4.69(\mathrm{~s}, 1 \mathrm{H}), 4.77(\mathrm{~s}, 1 \mathrm{H}), 6.61(\mathrm{~d}, J=8.2 \mathrm{~Hz}, 2 \mathrm{H}), 6.71(\mathrm{t}, J=7.4 \mathrm{~Hz}, 1 \mathrm{H}), 6.83(\mathrm{~d}$, $J=8.7 \mathrm{~Hz}, 1 \mathrm{H}), 7.1(\mathrm{t}, J=9.3 \mathrm{~Hz}, 2 \mathrm{H}), 7.0(\mathrm{~m}, 2 \mathrm{H}) ;{ }^{13} \mathrm{C} \mathrm{NMR}\left(\mathrm{CDCl}_{3}, 75.5 \mathrm{MHz}\right): \delta 53.83$, 54.71, 55.32, 56.7, 113.39, 113.46, 113.86, 118.58, 120.14, 129.2, 129.7, 137.24, 146.2, 159.9; Anal. Calcd for $\mathrm{C}_{17} \mathrm{H}_{22} \mathrm{NO}_{5} \mathrm{P}: \mathrm{C}, 58.12 ; \mathrm{H}, 6.27 ; \mathrm{N}, 3.99$. Found: C, 58.07; H, 6.15; N, 4.11.

Dimethyl $\boldsymbol{\alpha}$-anilino- $\boldsymbol{\alpha}$-(1-naphthyl)methylphosphonate (3m). Yield $208 \mathrm{mg}$; white solid; mp $132{ }^{\circ} \mathrm{C}$; IR (KBr): 3308, 3027, 1949, 1601, 1236, 1054, 1026, $830 \mathrm{~cm}^{-1} ;{ }^{1} \mathrm{H} \mathrm{NMR}\left(\mathrm{CDCl}_{3}, 300\right.$ MHz): $\delta 3.15(\mathrm{~d}, J=10.5 \mathrm{~Hz}, 3 \mathrm{H}), 3.81(\mathrm{~d}, J=10.8 \mathrm{~Hz}, 3 \mathrm{H}), 5.06(\mathrm{t}, J=8.7 \mathrm{~Hz}, 1 \mathrm{H}), 5.67(\mathrm{dd}, J$ $=7.7 \mathrm{~Hz}, 7.7 \mathrm{~Hz}, 1 \mathrm{H}), 6.55(\mathrm{~d}, J=8.1 \mathrm{~Hz}, 2 \mathrm{H}), 6.66(\mathrm{t}, J=7.3 \mathrm{~Hz}, 1 \mathrm{H}), 7.05(\mathrm{t}, J=7.9 \mathrm{~Hz}, 2 \mathrm{H})$, $7.45(\mathrm{t}, J=7.7 \mathrm{~Hz}, 1 \mathrm{H}), 7.55(\mathrm{t}, J=7.5 \mathrm{~Hz}, 1 \mathrm{H}), 7.63(\mathrm{t}, J=7.4 \mathrm{~Hz}, 1 \mathrm{H}), 7.79$ (d, $J=9.1 \mathrm{~Hz}$, $2 \mathrm{H}), 7.91(\mathrm{~d}, J=7.8 \mathrm{~Hz}, 1 \mathrm{H}), 8.23(\mathrm{~d}, J=8.7 \mathrm{~Hz}, 1 \mathrm{H}) ;{ }^{13} \mathrm{C} \mathrm{NMR}\left(\mathrm{CDCl}_{3}, 75.5 \mathrm{MHz}\right): \delta 50.2$, 52.2, 53.7, 113.6, 118.1, 118.4, 122.6, 125.5, 125.6, 125.7, 126.5, 128.6, 128.7, 129.2, 131.3, 131.4, 133.8, 145.9. Anal. Calcd for $\mathrm{C}_{19} \mathrm{H}_{20} \mathrm{NO}_{3} \mathrm{P}: \mathrm{C}, 66.86 ; \mathrm{H}, 5.86 ; \mathrm{N}, 4.10$. Found: C, 66.91; $\mathrm{H}, 5.83 ; \mathrm{N}, 4.06 \%$.

Dimethyl $\boldsymbol{\alpha}$-anilino- $\boldsymbol{\alpha}$-(ethyl)methylphosphonate (3o). Yield $340 \mathrm{mg}$; white crystals; mp $40{ }^{\circ} \mathrm{C}$; IR (KBr): 3432, 3029, 2373, 1629, 1456, 1236, 1040, $702 \mathrm{~cm}^{-1} ;{ }^{1} \mathrm{H} \mathrm{NMR}\left(\mathrm{CDCl}_{3}, 300 \mathrm{MHz}\right): \delta$ $1.03(\mathrm{t}, J=7.4 \mathrm{~Hz}, 3 \mathrm{H}), 1.62-1.76(\mathrm{~m}, 1 \mathrm{H}), 1.91-2.05(\mathrm{~m}, 1 \mathrm{H}), 2.15(\mathrm{~s}, 1 \mathrm{H}), 3.52(\mathrm{~d}, J=10.4 \mathrm{~Hz}$, $3 \mathrm{H}), 3.73(\mathrm{~d}, J=10.8 \mathrm{~Hz}, 3 \mathrm{H}), 6.65(\mathrm{~d}, J=8.1 \mathrm{~Hz}, 2 \mathrm{H}), 6.71(\mathrm{t}, J=7.2 \mathrm{~Hz}, 1 \mathrm{H}), 7.16(\mathrm{~d}, J=7.8$ $\mathrm{Hz}, 2 \mathrm{H}) ;{ }^{13} \mathrm{C} \mathrm{NMR}\left(\mathrm{CDCl}_{3}, 75.5 \mathrm{MHz}\right): \delta 10.6,23.9,51.1,52.6,53.6,113.2,118.2,129.3,147.0$. Anal. Calcd for $\mathrm{C}_{11} \mathrm{H}_{17} \mathrm{NO}_{3} \mathrm{P}: \mathrm{C}, 54.54 ; \mathrm{H}, 7.02 ; \mathrm{N}, 5.76$. Found: C, 54.39; H, 7.45; N, 5.67\%.

Dimethyl $\boldsymbol{\alpha}$-(2-methylanilino)- $\boldsymbol{\alpha}$-(phenyl)methylphosphonate (4b). Yield $238 \mathrm{mg}$; white powder; mp $48{ }^{\circ} \mathrm{C}$; IR (KBr): 3418, 3028, 2956, 1596, 1507, 1543, 1240, 1047, $832 \mathrm{~cm}^{-1} ;{ }^{1} \mathrm{H}$ NMR $\left(\mathrm{CDCl}_{3}, 300 \mathrm{MHz}\right): \delta 2.2(\mathrm{~s}, 3 \mathrm{H}), 3.40(\mathrm{~d}, J=10.5 \mathrm{~Hz}, 3 \mathrm{H}), 3.68(\mathrm{~d}, J=10.5 \mathrm{~Hz}, 3 \mathrm{H})$, 
$4.56($ br s, $1 \mathrm{H}), 4.71(\mathrm{~s}, 1 \mathrm{H}), 6.32(\mathrm{~d}, J=7.8 \mathrm{~Hz}, 1 \mathrm{H}), 6.57(\mathrm{t}, J=7.4 \mathrm{~Hz}, 1 \mathrm{H}), 6.87(\mathrm{t}, J=7.8$ $\mathrm{Hz}, 1 \mathrm{H}), 6.97(\mathrm{~d}, J=7.2 \mathrm{~Hz}, 1 \mathrm{H}), 7.17-7.28(\mathrm{~m}, 3 \mathrm{H}), 7.38(\mathrm{~d}, J=6.1 \mathrm{~Hz}, 2 \mathrm{H}) ;{ }^{13} \mathrm{C} \mathrm{NMR}\left(\mathrm{CDCl}_{3}\right.$, 75.5 MHz): $\delta$ 17.5, 53.8, 54.7, 56.7, 111.3, 118.2, 123.0, 126.9, 127.6, 128.0, 128.7, 130.2, 135.7, 144.0. Anal. Calcd for $\mathrm{C}_{16} \mathrm{H}_{20} \mathrm{NO}_{3} \mathrm{P}: \mathrm{C}, 62.95$; H, 6.58; N, 4.59. Found: C, 62.89; H, 6.55; N, $4.67 \%$.

Dimethyl $\boldsymbol{\alpha}$-(4-bromoanilino)- $\boldsymbol{\alpha}$-(phenyl)methylphosphonate (4e). Yield $304 \mathrm{mg}$; white powder; mp $60{ }^{\circ} \mathrm{C}$; IR (KBr): 3310, 3006, 2952, 1593, 1492, 1238, 1029, $818 \mathrm{~cm}^{-1}$; ${ }^{1} \mathrm{H}$ NMR $\left(\mathrm{CDCl}_{3}, 300 \mathrm{MHz}\right): \delta 3.38(\mathrm{~d}, J=10.5 \mathrm{~Hz}, 3 \mathrm{H}), 3.68(\mathrm{~d}, J=10.8 \mathrm{~Hz}, 3 \mathrm{H}), 4.66(\mathrm{dd}, J=7.8 \mathrm{~Hz}$, $7.8 \mathrm{~Hz}, 1 \mathrm{H}), 4.83(\mathrm{t}, J=8.6 \mathrm{~Hz}, 1 \mathrm{H}), 6.40(\mathrm{~d}, J=8.7 \mathrm{~Hz}, 2 \mathrm{H}), 7.1(\mathrm{~d}, J=8.7 \mathrm{~Hz}, 2 \mathrm{H}), 7.19-7.29$ $(\mathrm{m}, 3 \mathrm{H}), 7.37(\mathrm{~d}, J=7.5 \mathrm{~Hz}, 2 \mathrm{H}) ;{ }^{13} \mathrm{C} \mathrm{NMR}\left(\mathrm{CDCl}_{3}, 75.5 \mathrm{MHz}\right): \delta 53.8,54.7,56.7,110.3,115.5$, 127.4, 128.2, 128.8, 131.9, 135.0, 145.1. Anal. Calcd for $\mathrm{C}_{15} \mathrm{H}_{17} \mathrm{BrNO}_{3} \mathrm{P}: \mathrm{C}, 48.65 ; \mathrm{H}, 4.59 ; \mathrm{N}$, 3.78. Found: C, 48.72; H, 4.62; N, 3.69\%.

Dimethyl $\boldsymbol{\alpha}$-(3-nitroanilino)- $\boldsymbol{\alpha}$-(phenyl)methylphosphonate (4g). Yield $267 \mathrm{mg}$; bright yellow crystals; mp $130{ }^{\circ} \mathrm{C}$; IR (KBr): 3281, 3082, 2954, 1622, 1533, 1347, 1234, 1030, $819 \mathrm{~cm}^{-1} ;{ }^{1} \mathrm{H}$ NMR $\left(\mathrm{CDCl}_{3}, 300 \mathrm{MHz}\right): \delta 3.40(\mathrm{~d}, J=10.6 \mathrm{~Hz}, 3 \mathrm{H}), 3.72(\mathrm{~d}, J=10.6 \mathrm{~Hz}, 3 \mathrm{H}), 4.73(\mathrm{~m}, 1 \mathrm{H})$, $5.53(\mathrm{t}, J=8.6 \mathrm{~Hz}, 1 \mathrm{H}), 6.81(\mathrm{~d}, J=7.5 \mathrm{~Hz}, 1 \mathrm{H}), 7.17(\mathrm{~m}, 1 \mathrm{H}), 7.27(\mathrm{~m}, 3 \mathrm{H}), 7.42(\mathrm{~m}, 5 \mathrm{H}) ;{ }^{13} \mathrm{C}$ NMR $\left(\mathrm{CDCl}_{3}, 75.5 \mathrm{MHz}\right): \delta 53.7,54.1,56.4,108.0,113.0,119.4,127.8,128.4,128.9,129.7$, 134.5, 147.1, 149.2. Anal. Calcd for $\mathrm{C}_{15} \mathrm{H}_{18} \mathrm{~N}_{2} \mathrm{O}_{5} \mathrm{P}$ : C, 53.41; H, 5.34; N, 8.31. Found: C, 53.51; H, $5.11 ; \mathrm{N}, 8.39 \%$.

\section{Acknowledgements}

B.K. thanks UGC, New Delhi for the minor research project scheme in providing financial assistance and to the technical staff of the Organic Instrumentation Laboratory, Calcutta University, for instrumental analysis.

\section{References}

1. (a) Kabachnik, M. I.; Medved, T. Ya. Dokl. Akad. Nauk SSSR 1952, 83, 689. (b) Kabachnik, M. I.; Medved, T. Ya. Chem. Abstr. 1953, 47, 2724. (c) Fields, E. J. Am. Chem. Soc. 1952, 74, 1528. (d) For reviews see: Cherkasov, R. A.; Galkin, V. I. Russ. Chem. Rev. 1998, 67, 857.

2. Smith, A. B.; Yager, K. M.; Taylor, C. M. J. Am. Chem. Soc. 1995, 117, 10879.

3. (a) Atherton, F. R.; Hassall, C. H.; Lambert, R. W. J. Med. Chem. 1986, 29, 29. (b) Du, S.; Faiger, H.; Belakhov, V.; Baasov, T. Bioorg. Med. Chem. 1997, 7, 2671.

4. (a) Giannousis, P. P.; Barlett, P. A. J. Med. Chem. 1987, 30, 1603. (b) Grembecka, J.; Mucha, A.; Cierpicki, T.; Kafarski, P. J. Med. Chem. 1989, 32, 2461.

5. Atherton, F. R.; Hassall, C. H.; Lambert, R. W. J. Med. Chem. 1986, 29, 20. 
6. Zomova, A. M.; Molodykh, Zh. V.; Kudryavtseva, L. A.; Teplyakova, L. V.; Fedorov, S. V.; Ivanov, B. E. Pharm. Chem. J. 1986, 20, 774.

7. Jin, L. H.; Song, B. A.; Zhang, G. P.; Xu, R. Q.; Zhang, S. M.; Gao, X. W.; Hu, D. Y.; Song Yang, S. Bioorg. Med. Chem. Lett. 2006, 16, 1537.

8. (a) Maier, L. Phosphorus, Sulfur, Silicon Relat. Elem. 1990, 53, 43. (b) Maier, L.; Diel, P. J. Phosphorus, Sulfur, Silicon Relat. Elem. 1991, 57, 57.

9. (a) Ghosh, R.; Maiti, S.; Chakraborty, A.; Maiti, D. K. J. Mol. Catal. A: Chemical 2004, 210, 53. (b) Heydari, A.; Hamadi, H.; Pourayoubi, M. Catal. Commun. 2007, 8, 1224. (c) Ambica,; Kumar, S.; Taneja, S. C.; Hundal, M. S.; Kapoor, K. K. Tetrahedron Lett. 2008, 49, 2208. (d) Chandrasekhar, S.; Jaya Prakash, S.; Jagadeswar, V.; Narsihmulu, Ch. Tetrahedron Lett. 2001, 42, 5561. (e) Azizi, N.; Saidi, M. R. Tetrahedron 2003, 59, 5329. (f) Sarvari, M. H. Tetrahedron 2008, 64, 5459. (g) Sobhani, S.; Safaei, E.; Asadi, M.; Jalili, F. J. Organomet. Chem. 2008, 693, 3313. (h) Vahdat, S. M.; Baharfar, R.; Tajbakhsh, Heydari, A.; Baghbanian, S. S.; Khaskar, S. Tetrahedron Lett. 2008, 49, 6501. (i) Akbari, J.; Heydari, A. Tetrahedron Lett. 2009, 50, 4236. (j) Heydari, A.; Khaskar, S.; Tajbakhsh, M. Tetrahedron Lett. 2009, 50, 77. (k) Vinu, A.; Kalita, P.; Balasubramanian, V. V.; Oveisi, H.; Selvan, T.; Mano, A.; Chari, M. A.; Reddy, B. V. S. Tetrahedron Lett. 2009, 50, 7132. (1) Uziel, J.; Genet, J. P. Russ. J. Org. Chem. 1997, 33, 1521. (m) Ma, J.-A. Chem. Soc. Rev. 2006, 35, 630. (n) Kukhar, V. P.; Soloshonok, V. A.; Solodenko, V. A. Phosphorus, Sulfur, Silicon Relat. Elem. 1994, 92, 239. (o) Ordóñez, M.; Rojas-Cabrera, H.; Cativiela, C. Tetrahedron 2009, 65, 17. (p) Kukhar, V. P.; Hudson, H. R., Aminophosphonic and Aminophosphinic Acids: Chemistry and Biological Activity by Wiley: New York, 2000. (q) Kukhar, V. P.; Romanenko, V. D. Andrew B. Hughes, Chemistry of Aminophosphonic Acids and Phosphonopeptides, In Amino Acids, Peptides and Proteins in Organic Chemistry Wiley, Vol. 4; 2009. (r) Kudrimoti, S.; Bommena, V. R. Tetrahedron Lett. 2005, 46, 1209. (s) Mu, X. -J.; Lei, M.-Y.; Zou, J.-P.; Zhang, W. Tetrahedron Lett. 2006, 47, 1125. (t) Bhagat, S.; Chakraborti, S. J. Org. Chem. 2007, 72, 1263.

10. (a) Martinez, R.; Ramon, D. J.; Yus, M. Adv. Synth. Catal. 2008, 350, 1235. (b) Mukhopadhyay, C.; Rana, S. Catal. Commun. 2009, 11, 285. (c) Mannathusamy, G.; Purusothaman, S.; Jeyaraman, T.; Vijayakumar, K.; Muthuvel, E. R. Lett. Org. Chem. 2008, $5,142$.

11. (a) Karmakar, B.; Nayak, A.; Chowdhury, B.; Banerji, J. Arkivoc 2009, XII, 209. (b) Postole, G.; Chowdhury, B.; Karmakar, B.; Pinki, K.; Banerji, J.; Auroux, A. J. Catal. 2010, 269, 110. (c) Karmakar, B.; Chowdhury, B.; Banerji, J. Catal. Commun. 2010, 11, 601. (d) Karmakar, B.; Banerji, J. Tetrahedron Lett. 2010, 51, 3855. (e) Karmakar, B.; Sinhamahapatra, A.; Panda, A. B.; Banerji, J.; Chowdhury, B. Appl. Catal. A: Gen. 2010, Article in Press, doi: 10.1016/j.apcata.2010.10.030.

12. (a) Li, J.; Hao, Y.; Li, H.; Xia, M.; Sun, X.; Wang, L. Micropor. Mesopor. Mater. 2009, 120, 421. (b) Mong, M. S.; Jeng, E. S.; Ying, J. Y. Nano Lett. 2001, 1, 637. (c) Liu, S.; Zhang, X.; Li, J.; Wei, W.; Sun, Y. Catal Commun. 2008, 9, 1527. 